\title{
The Association of Hepatitis B Vaccine Supply Policy with Timing of Receipt of the First Dose of Hepatitis B Vaccination
}

\author{
Zhen Zhao', Trudy V. Murphy ${ }^{2}$ \\ ${ }^{1}$ National Center for Immunization and Respiratory Diseases, Centers for Disease Control and Prevention, Atlanta, USA \\ ${ }^{2}$ National Center for HIV/AIDS, Viral Hepatitis, STD\&TB Prevention, Centers for Disease Control and Prevention, Atlanta, USA \\ Email: zzhao@cdc.gov
}

Received July 11, 2012; revised August 14, 2012; accepted August 26, 2012

\begin{abstract}
An estimated 800,000 - 1.4 million persons in the US have chronic Hepatitis B Virus (HBV) infection. The risk for chronic infection is greatest among young children; approximately $90 \%$ of infants will remain chronically infected with HBV. Approximately $25 \%$ of those who become chronically infected during childhood die prematurely from cirrhosis or liver cancer. Hepatitis B vaccination is the most effective measure to prevent HBV infection and its consequences. In 2006, 29 US states had Hepatitis B Vaccine Supply (HBVS) policy which either supplies hepatitis B vaccine at no cost to all providers for all children or provides hepatitis B vaccine to delivery hospitals-only free of charge for all infants; other 21 US states and the District of Columbia did not have. 17,636 infants born in 2006 obtained from 2007-2009 National Immunization Survey (NIS) were analyzed with survival analysis procedures of Kaplan-Meier estimate and Cox proportional hazards model for complex sample survey to evaluate the association between state HBVS policy and the timing of infant age in days to receipt of hepatitis B vaccination. State HBVS policy is associated with infant age in days from birth to receipt of the first dose of hepatitis $\mathrm{B}$ vaccine $(\mathrm{P}<0.01)$, and to completion of the 3-dose hepatitis $\mathrm{B}$ vaccine series $(\mathrm{P}<0.01)$. Receipt of the first dose of hepatitis $\mathrm{B}$ vaccine occurred $31 \%$ earlier among infants residing in states with HBVS policy than among infants residing in states without (adjusted hazards ratio $1.31,95 \% \mathrm{CI}(1.23,1.39)$ ). Completion of the 3 -dose hepatitis B vaccine series were $12 \%$ sooner among infants living in states with HBVS policy than among infants living in states without (adjusted hazards ratio $1.12,95 \% \mathrm{CI}(1.06,1.18)$ ). State HBVS policy may help overcome barriers to timely delivery of hepatitis B vaccines to infants.
\end{abstract}

Keywords: First Dose Hepatitis B Vaccine; 3-Dose Hepatitis B Vaccine Series; Vaccine Supply Policy; Vaccination Coverage; Cox Proportional Hazards Model

\section{Introduction}

An estimated 800,000 - 1.4 million persons in the US have chronic hepatitis B virus (HBV) infection. The risk for chronic infection is greatest among young children; approximately $90 \%$ of infants will remain chronically infected with HBV. By contrast, about $95 \%$ of adults recover completely from HBV infection and do not become chronically infected. Approximately $25 \%$ of those who become chronically infected during childhood die prematurely from cirrhosis or liver cancer [1]. Hepatitis $B$ vaccination is the most effective measure to prevent HBV infection and its consequences. Completion of hepatitis B immunization alone, when started within the first day of life, prevents $70 \%-95 \%$ of perinatal HBV infections, as well as early childhood HBV infections acquired from HBV-infected household contacts [2]. In 1991, the Advisory Committee on Immunization Prac- tices (ACIP) proposed, for the first time, that hepatitis B vaccination be recommended for all infants regardless of the hepatitis B surface antigen ( $\mathrm{HBsAg}$ ) status of the mother [3]. The preferred vaccination schedule of the ACIP and the American Academy of Pediatrics (AAP) calls for administering the first dose of hepatitis B vaccine during the newborn period, before the infant is discharged from the hospital; the second dose at age 1 to 2 months; and the third dose at age 6 to 18 months [3-5]. The critical feature of the recommendation is to initiate the 3-dose hepatitis B vaccine series at birth.

In the US, a state vaccine financing policy describes which groups of children and in which settings the state will purchase vaccine with Vaccines for Children (VFC) program funds, Public Health Service Section 317 Immunization Grants Program funds, or state funds. The state vaccine financing policies range from very com- 
prehensive approaches, such as a Universal vaccine financing policy, in which the state provides all vaccines to all providers for all children, to approach in which the state only provides vaccines for those children who are VFC eligible [6,7].

The uptake of heptavalent pneumococcal conjugate vaccine (PCV7) among children aged 19 to 35 months residing in the United States and how uptake rates differed by state vaccine financing policy were evaluated with logistic regression [7].

The research letter [8] suggested that the median of hepatitis $\mathrm{B}$ birth dose vaccination rate might be higher for infants with than without specific hospital hepatitis B vaccine supply policy. The birth dose of hepatitis $B$ vaccination status (yes vs. no) was defined as the first dose of hepatitis B vaccine administered within 2 days of life for infants born in 2002. Due to the data limitation, nonparametric Wilcoxon rank-sum test was applied to compare the median of hepatitis B birth dose vaccination rates between the two independent groups. Thus that analysis couldn't account for the critical timing information of infant age in days from birth to receipt of the first dose of hepatitis B vaccine, and might compromise the precision of the estimate and inference for the effect of specific hospital hepatitis B vaccine supply policy. In addition, the association of specific hospital hepatitis B vaccine supply policy with completion of the 3-dose hepatitis B vaccine series was not evaluated.

Robust data were lacking with regard to the association between timing of infants age in days to receipt of hepatitis B vaccination and state Hepatitis B Vaccine Supply (HBVS) policy which will be defined in Section 2.2. In this study, we conducted a birth year cohort study with infants born in 2006 obtained from 2007-2009 National Immunization Survey (NIS). The primary objective of this study is to evaluate the association of state HBVS policy with timing of infant age in days from birth to receipt of the first dose of hepatitis $B$ vaccine. The secondary objective is to assess whether state HBVS policy is associated with earlier completion of the 3-dose hepatitis $\mathrm{B}$ vaccine series. In order to achieve the above two objectives, survival analysis for complex sample survey data was applied in this study.

\section{Materials and Methods}

\subsection{Data Resource}

A cohort of infants born in 2006 in the US was analyzed in this study. Information about this birth cohort was obtained from NIS conducted during 2007-2009. The NIS is carried out annually by the Centers for Disease Control and Prevention (CDC) to acquire national, state, and selected urban-area estimates of vaccination coverage for the US non-institutionalized population of chil- dren age 19 - 35 months [9]. For NIS 2007-2009 data, the overall household response rates based on Council of American Survey and Research Organizations (CASRO) guidelines ranged from $63.2 \%$ to $64.9 \%$ [10]. The analyses in this study were limited to children with adequate provider data, children for whom sufficient vaccination history information is obtained from their providers to determine whether they are up-to-date with respect to the recommended vaccination schedule, the proportions of children with adequate provider data ranged from $85.4 \%$ to $87.6 \%$ [10]. In the 2006 birth cohort, there were 17,636 infants that represented approximate 4.3 million infants born in the US in 2006.

The socio-demographic factors related to infants, family, mother, and vaccination provider available in the NIS were examined in both univariate and multivariable analyses. Those factors have previously been found to be associated with infant vaccination coverage in the United States [11-13]: infant race/ethnicity (Non-Hispanic White, Non-Hispanic Black, Hispanic, other), first born status (yes vs. no), and number of siblings ( 0 vs. $\geq 1$ ); family poverty status (at or above vs. below poverty level), and locality (urban, suburban, rural); mother's education level ( $\leq 12$ years vs. $>12$ years), marital status (married vs. not married), and mother's age ( $\leq 29$ years vs. $\geq 30$ years); number ( 1 vs. $\geq 2$ ) and type (public, private, other) of vaccination providers; and survey interview language (English, Spanish, or other).

\subsection{State Hepatitis B Vaccine Supply (HBVS) Policy}

In the US, a state vaccine financing policy was self-reported by the state immunization program to the National Center for Immunization and Respiratory Diseases, Centers for Disease Control and Prevention. The states were defined by their vaccine financing policy category: Universal; Universal-Select; VFC and Underinsured; VFC and Underinsured-Select; VFC-Only; and Other [6,7,14]. The states with Universal or Universal-Select policy supply hepatitis B vaccine among others at no cost to all vaccination providers for all children, in 2006 sixteen states were in this category (Alaska, New Hampshire, New Mexico, Rhode Island, Vermont, Washington, Connecticut, Hawaii, Idaho, Maine, Massachusetts, Nevada, North Carolina, North Dakota, South Dakota, and Wyoming). In addition, some states have established a specific hospital hepatitis B vaccine supply policy that provides hepatitis $B$ vaccine to delivery hospitals-only free of charge for all infants regardless of the eligibility for VFC or the health insurance status of infants [8]. In 2006, thirteen states were in this category (Arizona, Arkansas, Kansas, Kentucky, Louisiana, Michigan, Montana, New York, Ohio, Oregon, Pennsylvania, Utah, and Wisconsin). 
Data on state specific hospital hepatitis B vaccine supply policy were obtained from CDC's Perinatal Hepatitis B Prevention Program Annual Assessment Report for year 2006; this is the most recent information available on state specific hospital hepatitis B vaccine supply policy.

To explore the association of hepatitis B vaccine supply policy with infant hepatitis B vaccination, in this study, the preceding two types of policies together are defined as state Hepatitis B Vaccine Supply (HBVS) policy. Thus, in 2006 in the US, 29 US states had state HBVS policy; and 21 US states and the District of Columbia did not have state HBVS policy.

\subsection{Statistical Analyses}

Survival analysis procedures for complex sample survey [15] were adopted to evaluate the association of state HBVS policy defined above with timing of infant hepatitis B vaccination. In the primary analysis, the "time" dependent variable was defined as infant age in days from birth to receipt of the first dose of hepatitis $B$ vaccine. The unprecedented feature of the recommended hepatitis $B$ vaccination schedule by ACIP and AAP is to initiate hepatitis B vaccine series at birth, thus in this study if infant age in days from birth to the first dose of hepatitis $B$ vaccination was less than or equal to 30 days then this age was defined as an event, otherwise the age was right censored at 30 days $[5,16]$. As for completion of the 3-dose hepatitis B vaccine series, by the recommended schedule the third dose of hepatitis B vaccine should be administered at least 112 days (16 weeks) after the first dose and completed by 549 days (18 months) of age. Thus in the secondary analysis, infants whose age being greater than or equal to 112 days were included, and if infant age in days from birth to the third dose of hepatitis $B$ vaccination was less than or equal to 549 days, then the age was defined as an event, otherwise the age was right censored at 549 days.

The two cumulative vaccination coverage curves for the first dose of hepatitis B vaccination were estimated with the use of Kaplan-Meier model by infant age in days and stratified by state HBVS policy status (yes vs. no), and were compared with the use of log-rank test. Similarly, the cumulative vaccination coverage curve estimation and comparison for completion of the 3-dose hepatitis B vaccine series applied. The Cox proportional hazards model $[17,18]$ was applied to evaluate the association of state HBVS policy with infant age in days from birth to receipt of the first dose of hepatitis B vaccine, also to completion of the 3-dose hepatitis B vaccine series, controlling for the selected socio-demographic factors. The backward-selection method $[19,20]$ was applied to obtain the final model which contains the significant predictors. The proportional hazards assumptions of Cox model for NIS 2006 birth cohort data were ex- amined by the graphic method, and were found to be valid for all factors investigated.

\section{Results}

\subsection{Prevalence Rates of State HBVS Policy}

The prevalence rates of state HBVS policy across all strata of the significant socio-demographic factors, which were obtained in the final Cox model for evaluating the association of state HBVS policy with infant age in days from birth to receipt of the first dose of hepatitis B vaccine controlling for the selected socio-demographic factors, ranged from $26.9 \%$ to $46.9 \%$, and the national rate was $38.8 \%$, as presented in Table $\mathbf{1}$. The prevalence rate for Non-Hispanic White infants, $46.9 \%$, was the highest among all segments of race/ethnicity factor, and it was significantly higher $(p<0.05)$ than the prevalence rate for Non-Hispanic Black infants. The prevalence rate for Hispanic infants, $26.9 \%$, was the lowest $(p<0.05)$ among all groups in race/ethnicity factor. Factor race/ ethnicity was significantly associated with state HBVS policy status $(p<0.01)$. Infants whose mother's education $>12$ years were more likely than those whose mother's education $\leq 12$ years to be residing in states with HBVS policy $(p<0.01)$. Infants who had 1 vaccination provider were more likely than those with $\geq 2$ vaccination providers to live in HBVS policy states $(p=$ $0.02)$.

\subsection{The Association of State HBVS Policy with the First Dose of Hepatitis B Vaccination}

Two curves for the first dose of hepatitis B cumulative vaccination coverage by infant age in days stratified by state HBVS policy status were shown in Figure 1. Overall, the cumulative vaccination coverage for the first dose of hepatitis B vaccine was significantly higher for infants living in states with HBVS policy than infants living in states without such policy ( $p$-value $<0.01$ by the logrank test). Table 2 presented the hepatitis B first dose cumulative vaccination coverage with $95 \%$ confidence interval at infant age 0 through 3 days, and 1 through 4 weeks for all infants and stratified by state HBVS policy status. At each specific infant age, infants residing in states with HBVS policy would be most likely ( $p$-value $<$ $0.05)$ to receive the first dose of hepatitis $B$ vaccine compared to infants residing in states without such policy.

The main effect of state HBVS policy was significantly associated with infant age in days from birth to receipt of the first dose of hepatitis B vaccine ( $p$-value $<$ 0.01 , F-test) controlling for the six significant covariates acquired from the Cox model. Receipt of the first dose of hepatitis B vaccine occurred significantly earlier among infants living in states with HBVS policy than among 
Table 1. Prevalence rates of state Hepatitis B Vaccine Supply (HBVS) policy by selected socio-demographic factors.

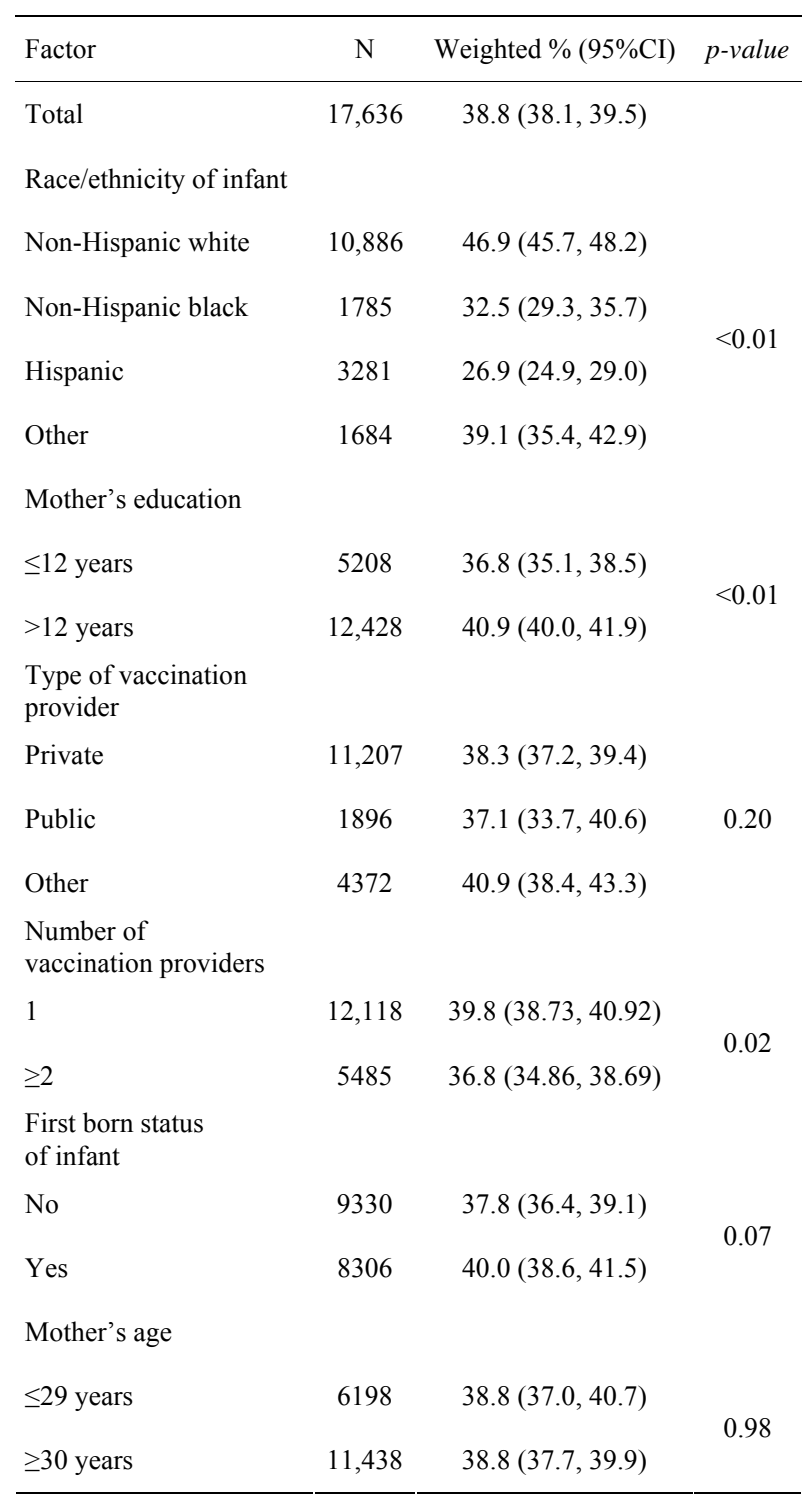

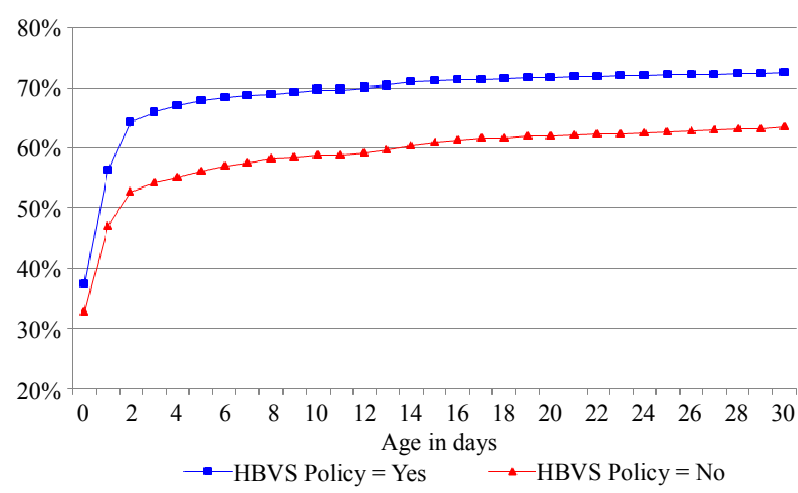

Figure 1. Cumulative vaccination coverage in percentage for the first dose of Hepatitis B (HepB) vaccine by infant age in days stratified by state Hepatitis B Vaccine Supply (HBVS) policy status.
Table 2. Cumulative vaccination coverage for the first dose of Hepatitis B (HepB) vaccine by infant age in days/weeks stratified by state Hepatitis B Vaccine Supply (HBVS) policy status.

\begin{tabular}{cccc}
\hline $\begin{array}{c}\text { Infant } \\
\text { Age }\end{array}$ & $\begin{array}{c}\text { All Infants } \\
\%(95 \% \mathrm{CI})\end{array}$ & $\begin{array}{c}\text { HBVS Policy }=\text { Yes } \\
\%(95 \% \mathrm{CI})\end{array}$ & $\begin{array}{c}\text { HBVS Policy }=\text { No } \\
\%(95 \% \mathrm{CI})\end{array}$ \\
\hline 0 day & $34.5(33.3,35.8)$ & $37.4(35.8,39.0)$ & $32.7(30.9,34.5)$ \\
1 day & $50.6(49.3,51.9)$ & $56.3(54.7,57.9)$ & $47.0(45.1,48.9)$ \\
2 days & $57.2(55.9,58.5)$ & $64.4(62.8,65.9)$ & $52.7(50.8,54.6)$ \\
3 days & $58.7(57.4,60.0)$ & $65.8(64.3,67.4)$ & $54.2(52.3,56.1)$ \\
1 week & $61.9(60.6,63.2)$ & $68.9(67.4,70.3)$ & $57.5(55.7,59.4)$ \\
2 weeks & $64.5(63.2,65.8)$ & $70.9(69.4,72.3)$ & $60.4(58.6,62.3)$ \\
3 weeks & $65.9(64.6,67.1)$ & $71.8(70.3,73.2)$ & $62.1(60.3,64.0)$ \\
4 weeks & $66.7(65.4,67.9)$ & $72.3(70.8,73.7)$ & $63.2(61.3,65.0)$ \\
\hline
\end{tabular}

infants living in states without such policy (adjusted hazards ratio $1.31,95 \% \mathrm{CI}(1.23,1.39))$; significantly earlier among Non-Hispanic Black infants than among NonHispanic White infants (adjusted hazards ratio 1.16, $95 \%$ CI $(1.04,1.29))$; and significantly sooner among infants having multiple vaccination providers than among infants having only 1 vaccination provider (adjusted hazards ratio $1.25,95 \% \mathrm{CI}(1.17,1.35))$ as presented in Table 3 . In addition, the result acquired by unadjusted Cox model were consistent with the result obtained from the adjusted Cox model with regard to the significance status of state HBVS policy measured by hazards ratios.

\subsection{The Association between Completion of the 3-Dose Hepatitis B Vaccine Series and State HBVS Policy}

The two cumulative vaccination coverage curves for completion of the 3-dose hepatitis B vaccine series by infant age in days stratified by state HBVS policy status were estimated and compared. In general, the cumulative vaccination coverage for completion of the 3-dose hepatitis B vaccine series was significantly higher among infants living in states with HBVS policy than among infants living in states without such policy ( $p$-value $<$ 0.01 by the log-rank test).

The main effect of state HBVS policy was significantly associated with infant age in days from birth to completion of the 3-dose hepatitis $\mathrm{B}$ vaccine series ( $p$-value $<0.01, \mathrm{~F}$-test) controlling for other six significant predictors obtained from the Cox model. The adjusted hazards ratio for state HBVS policy 1.12, with $95 \% \mathrm{CI}(1.06$, 1.18), suggested that completion of the 3-dose hepatitis $B$ vaccine series was significantly sooner among infants residing in states with HBVS policy than among infants residing in states without such policy. Infants who 
Table 3. Hazards Ratio (HR) of infant age in days from birth to receipt of the first dose of hepatitis $B$ vaccine between strata of state Hepatitis B Vaccine Supply (HBVS) policy and the significant socio-demographic factors.

\begin{tabular}{|c|c|c|}
\hline Factors & $\begin{array}{l}\text { Unadjusted } \\
\text { HR } \\
(95 \% \mathrm{CI})\end{array}$ & $\begin{array}{l}\text { Multivariable } \\
\text { Adjusted HR } \\
(95 \% \mathrm{CI})\end{array}$ \\
\hline \multicolumn{3}{|l|}{$\begin{array}{l}\text { State Hepatitis B Vaccine Supply } \\
\text { (HBVS) policy status }\end{array}$} \\
\hline Yes & $\begin{array}{l}1.28 \\
(1.20,1.36)\end{array}$ & $\begin{array}{l}1.31 \\
(1.23,1.39)\end{array}$ \\
\hline No & 1.00 & 1.00 \\
\hline \multicolumn{3}{|l|}{ Race/ethnicity of infant } \\
\hline Non-Hispanic white & 1.00 & 1.00 \\
\hline Non-Hispanic black & $\begin{array}{l}1.14 \\
(1.02,1.26)\end{array}$ & $\begin{array}{l}1.16 \\
(1.04,1.29)\end{array}$ \\
\hline Hispanic & $\begin{array}{l}1.09 \\
(1.00,1.18)\end{array}$ & $\begin{array}{l}1.09 \\
(1.00,1.19)\end{array}$ \\
\hline Other & $\begin{array}{l}1.15 \\
(1.03,1.29)\end{array}$ & $\begin{array}{l}1.17 \\
(1.05,1.30)\end{array}$ \\
\hline \multicolumn{3}{|l|}{ Mother's education } \\
\hline$\leq 12$ years & $\begin{array}{l}1.13 \\
(1.06,1.21)\end{array}$ & $\begin{array}{l}1.08 \\
(1.01,1.16)\end{array}$ \\
\hline$>12$ years & 1.00 & 1.00 \\
\hline \multicolumn{3}{|l|}{ Type of vaccination providers } \\
\hline Private & $\begin{array}{l}1.01 \\
(0.89,1.13)\end{array}$ & $\begin{array}{l}1.09 \\
(0.96,1.23)\end{array}$ \\
\hline Public & 1.00 & 1.00 \\
\hline Other & $\begin{array}{l}1.19 \\
(1.05,1.36)\end{array}$ & $\begin{array}{l}1.16 \\
(1.02,1.32)\end{array}$ \\
\hline \multicolumn{3}{|l|}{ Number of vaccination providers } \\
\hline 1 & 1.00 & 1.00 \\
\hline$\geq 2$ & $\begin{array}{l}1.29 \\
(1.20,1.38)\end{array}$ & $\begin{array}{l}1.25 \\
(1.17,1.35)\end{array}$ \\
\hline \multicolumn{3}{|l|}{ First born status of infant } \\
\hline No & 1.00 & 1.00 \\
\hline Yes & $\begin{array}{l}0.96 \\
(0.90,1.03)\end{array}$ & $\begin{array}{l}0.94 \\
(0.88,1.00)\end{array}$ \\
\hline \multicolumn{3}{|l|}{ Mother's age } \\
\hline$\leq 29$ years & $\begin{array}{l}1.16 \\
(1.08,1.24)\end{array}$ & $\begin{array}{l}1.11 \\
(1.03,1.19)\end{array}$ \\
\hline$\geq 30$ years & 1.00 & 1.00 \\
\hline
\end{tabular}

had 0 sibling were significantly earlier than infants who had 1 or more siblings with respect to completion of the 3-dose hepatitis B vaccine series (adjusted hazards ratio $1.13,95 \%$ CI $(1.06,1.21))$. Also, the significance status of state HBVS policy gained by unadjusted Cox model, (hazards ratio $1.11,95 \% \mathrm{CI}(1.05,1.17)$ ), was consistent with the result achieved from the adjusted Cox model, (hazards ratio $1.12,95 \% \mathrm{CI}(1.06,1.18)$ ).

\section{Discussion}

In our current study, both the unadjusted and adjusted analyses showed the consistent conclusions: infant age in days from birth to receipt of the first dose of hepatitis B vaccine was significantly associated with state HBVS supply policy, hazards ratios are $1.28(1.20,1.36)$ and $1.31(1.23,1.39)$ respectively. Similarly, consistent and significant results were obtained with regard to the association between infant age in days from birth to completion of the 3-dose hepatitis $B$ vaccine series and state HBVS policy, hazards ratios are $1.11(1.05,1.17)$ and $1.12(1.06,1.18)$ for unadjusted and adjusted analysis separately. These consistent results suggested that the association between hepatitis B vaccination and state HBVS policy is both positive and robust.

This study suggested that state HBVS policy was significantly associated with infant age in days from birth to receipt of the first dose of hepatitis B vaccine. Infants residing in states with HBVS policy received the first dose of hepatitis B vaccine significantly early than those residing in states without such policy. For example, $56.3 \%$ of infants with state HBVS policy received the first dose of hepatitis B vaccine by age 1 day; however $56.0 \%$ of infants without state HBVS policy received the first dose of hepatitis B vaccine by age 5 days. Similarly, infants completed the 3-dose of hepatitis B vaccine series significantly sooner among infants with state HBVS policy than among infants without such policy. For instance, $82.8 \%$ of infants with state HBVS policy completed the 3-dose hepatitis B vaccine series by age 10 months; but $82.8 \%$ of infants without such policy completed the 3-dose hepatitis B vaccine series by age 11.3 months.

This study showed that the first dose of hepatitis B vaccine was administered significantly earlier among infants residing in states with HBVS policy, which is important. However, it is also important to know if state HBVS policy is associated with earlier completion of the 3-dose hepatitis B vaccine series. As the secondary objective, this study suggested that state HBVS policy is associated with earlier completion of the 3-dose hepatitis $B$ vaccine series. The significant and positive answer strengthens the argument for state HBVS supply policy.

Some potential limitations of this study were that the NIS used the random-digit-dial residential landline telephone survey and has experienced under-representation due to non-response and wireless telephone use. Although the survey has been adjusted to account for these changes, under-representation of households could increase the potential bias in survey estimates. Also, nonresponse bias after weighting adjustments and possible incomplete ascertainment of hepatitis B vaccination by provider record check may cause additional bias. Finally, a few sub-state estimation areas rotated in or out in the survey design during the last few years. However a recent study [21] suggested that the total survey error in the NIS may be small with a mean of $1.7 \%, 95 \%$ CI $(1.71 \%$, 
$1.74 \%$ ), and would likely not have changed the conclusions in this study.

\section{Conclusion}

State HBVS policy has significantly improved access to hepatitis B vaccine for infants in the US. This policy results in earlier first dose of hepatitis B vaccination, such as before discharge from the delivery hospital or birthing center, sooner completion of the 3-dose hepatitis B vaccine series, and higher hepatitis B vaccination coverage. State HBVS policy may help overcome barriers to timely delivery of hepatitis B vaccines to infants.

\section{Disclaimer}

The findings and conclusions in this article are solely the responsibility of the authors and do not necessarily represent the official views of Centers for Disease Control and Prevention.

\section{REFERENCES}

[1] Centers for Disease Control and Prevention, "Hepatitis B FAQs for Health Professional," 2012. http://www.cdc.gov/hepatitis/hbv/HBVfaq.htm

[2] Centers for Disease Control and Prevention, "A Comprehensive Immunization Strategy to Eliminate Transmission of Hepatitis B Virus Infection in the United States: Recommendations of the Advisory Committee on Immunization Practices (ACIP) Part 1: Immunization of Infants, Children, and Adolescents," Morbidity and Mortality Weekly Report, Vol. 54, No. RR-16, 2005, pp. 1-23.

[3] Centers for Disease Control and Prevention, "Hepatitis B virus: A Comprehensive Strategy for Eliminating Transmission in the United States through Universal Childhood Vaccination: Recommendations of the Immunization Practices Advisory Committee (ACIP)," Morbidity and Mortality Weekly Report, Vol. 40, No. RR-13, 1991, pp. 1-19.

[4] American Academy of Pediatrics Committee on Infectious Diseases, "Universal Hepatitis B Immunization," Pediatrics, Vol. 89, No. 4, 1992, pp. 795-800.

[5] Centers for Disease Control and Prevention, "Recommended Immunization Schedule for Persons Aged 0 through 6 years_-United States," 2012.

http://www.cdc.gov/vaccines/recs/schedules/downloads/c hild/0-6yrs-schedule-pr.pdf

[6] G. M. Lee, J. M. Santoli, C. Hannan, M. L. Messonnier, J. E. Sabin, D. Rusinak, et al., "Gaps in Vaccine Financing for Underinsured Children in the United States," Journal of the American Medical Association, Vol. 298, No. 6, 2007, pp. 638-643. doi:10.1001/jama.298.6.638

[7] S. Stokley, K. M. Shaw, L. E. Barker, J. M. Santoli and A. Shefer, "Impact of State Vaccine Financing Policy on Uptake of Heptavalent Pneumococcal Conjugate Vaccine," American Journal of Public Health, Vol. 96, No. 7, 2006, pp. 1308-1313. doi:10.2105/AJPH.2004.057810
[8] L. Jacques-Carroll, S. Wang, Z. Zhao, T. Malik and F. David, "Hepatitis B Vaccination Coverage in Newborns and Vaccine Supply," Archives of Pediatrics \& Adolescent Medicine, Vol. 163, No. 5, 2009, p. 489.

doi:10.1001/archpediatrics.2009.22

[9] Centers for Disease Control and Prevention, "National and State Vaccination Coverage among Children 19-35 Months, United States 2010," Morbidity and Mortality Weekly Report, Vol. 64, No. 34, 2011, pp. 1157-1163.

[10] NORC at the University of Chicago, "National Immunization Survey Quarterly Report," 2011.

[11] E. T. Luman, A. E. Fiore, T. W. Strine and L. E. Barker, "Impact of Thimerosal-Related Changes in Hepatitis B Vaccine Birth-Dose Recommendations on Childhood Vaccination Coverage," Journal of the American Medical Association, Vol. 291, No. 19, 2004, pp. 2351-2358. doi:10.1001/jama.291.19.2351

[12] T. A. Santibanez, J. M. Santoli, C. B. Bridges and G. L. Euler, "Influenza Vaccination Coverage of Children Aged 6 to 23 Months: The 2002-2003 and 2003-2004 Influenza Seasons," Pediatrics, Vol. 118, No. 3, 2006, pp. 11671175. doi:10.1542/peds.2006-0831

[13] Z. Zhao and E. T. Luman, "Progress toward Eliminating Disparities in Vaccination Coverage among US Children, 2000-2008," American Journal of Preventive Medicine, Vol. 38, No. 2, 2010, pp. 127-137. doi:10.1016/j.amepre.2009.10.035

[14] Centers for Disease Control and Prevention, "The National Center for Immunization and Respiratory Diseases, Annual Progress Report, VFC Program Management Survey." http://www.cdc.gov/vaccines/programs/vfc

[15] Research Triangle Institute, "SUDAAN Language Manual, Release 10.0.1., Research Triangle Park, NC,” 2008.

[16] D. S. Lauderdale, R. J. Oram, K. P. Goldstein and R. S. Daum, "Hepatitis B Vaccination among Children in Inner-City Public Housing, 1991-1997," Journal of the American Medical Association, Vol. 282, No. 18, 1999 , pp. 1725-1730. doi:10.1001/jama.282.18.1725

[17] D. A. Binder, "Fitting Cox's Proportional Hazards Models from Survey Data," Biometrika, Vol. 79, No. 1, 1992, pp. 139-147. doi:10.1093/biomet/79.1.139

[18] D. Y. Lin, “On Fitting Cox's Proportional Models to Survey Data," Biometrika, Vol. 87, No. 1, 2000, pp. 37-47. doi:10.1093/biomet/87.1.37

[19] D. Hosmer and S. Lemeshow, "Applied Survival Analysis: Regression Modeling of Time to Event Data. Vol. 1," John Wiley \& Sons, New York, 1999.

[20] G. Thabut, J. D. Christic, W. K. Kremers, M. Fourbier and S. D. Halpern, "Survival Differences Following Lung Transplantation among US Transplant Centers," Journal of the American Medical Association, Vol. 304, No. 1, 2010, pp. 53-60.

[21] N. M. Molinari, K. M. Wolter, B. Skalland, R. Montgomery, M. Khare, P. J. Smith, et al., "Quantifying Bias in a Health Survey: Modeling Total Survey Error in the National Immunization Survey," Statistics in Medicine, Vol. 30, No. 5, 2011, pp. 505-514. 